\title{
Similarity Pattern Discovery for COVID-19 Using Sequential Decision Tree
}

\author{
Ziyuan $\operatorname{Li}^{\star}$ \\ Washington University in St. Louis, 6300 Forsyth Blvd, St. \\ Louis, MO 63105
}

\begin{abstract}
Decision tree algorithm that handles single classes is often used in biology and biomedicine. However, biological datasets frequently involve multiple classes related by a hierarchy, and each class can contain multiple labels, which cannot be predicted by the commonly used decision tree algorithm as classes used to define one higher hierarchical level would not appear again in the lower one. In this paper, a novel sequential decision tree (SDT) algorithm for hierarchical multi-label classification is proposed, and the SDT application to COVID-19 is examined. Comparing to the existing algorithms, SDT has good interpretability to be used on the population-demographicCOVID-19 (PDC) dataset. The goal of research is to compute the similarity of countries under the influence of COVID-19 using PDC as an input dataset. The hypothesis is made that the most similar countries predicted from the PDC dataset, which is defined by the DT leaves branched from the same end node, also have similar COVID-19 evolutions. It is defined by the small difference in infected, recovered, and dead time series curves of the countries. From data and statistical analysis, the hypothesis is supported.
\end{abstract}

\section{CCS CONCEPTS}

- Applied computing $\rightarrow$ Life and medical sciences; Health informatics.

\section{KEYWORDS}

Hierarchical multi-label classification, COVID-19, sequential decision tree

\section{ACM Reference Format:}

Ziyuan Li and Feng Liang. 2021. Similarity Pattern Discovery for COVID19 Using Sequential Decision Tree. In 2021 11th International Conference on Biomedical Engineering and Technology (ICBET '21), March 17-20, 2021, Tokyo, Japan. ACM, New York, NY, USA, 9 pages. https://doi.org/10.1145/ 3460238.3460250

\section{INTRODUCTION}

It is acknowledged that COVID-19 is a highly transmissible disease and associated with acute respiratory syndrome. The World Health Organization (WHO) identified the COVID-19 outbreak as a

*First Author's Email: arthurli@wustl.edu

Permission to make digital or hard copies of all or part of this work for personal or classroom use is granted without fee provided that copies are not made or distributed for profit or commercial advantage and that copies bear this notice and the full citation on the first page. Copyrights for components of this work owned by others than ACM must be honored. Abstracting with credit is permitted. To copy otherwise, or republish, to post on servers or to redistribute to lists, requires prior specific permission and/or a fee. Request permissions from permissions@acm.org.

ICBET '21, March 17-20, 2021, Tokyo, Japan

(C) 2021 Association for Computing Machinery.

ACM ISBN 978-1-4503-8789-7/21/03 . \$ \$15.00

https://doi.org/10.1145/3460238.3460250

\author{
Feng Liang \\ Beijing Normal University, No.19 Xinjiekouwai Street, \\ Haidian District, Beijing 100875, China
}

world-wide pandemic [12]. Until April 5, 2020, WHO has reported 113820168 confirmed cases globally [3]. Since different countries implement different measures to contain the transmission like early "lockdown" of cities in China [11] and the practice of social distancing in the U.S. [4], the pattern of the COVID-19 evolutions tends to be different as well. Based on the big data available about these countries, the underlying patterns can be uncovered.

Discovering the underlying patterns of the COVID-19 evolutions in different countries can facilitate subsequent investigations that what procedures in common can be taken by the countries with similar patterns to control the disease, as well as how a more accurate prediction on death rate or disease ending time can be obtained, especially for the countries without sufficient time-series data but with the patterns similar to other countries that have reliable time-series data [8]. Nonetheless, no relevant research was published to uncover the patterns, which is a prerequisite for more profound research. As a result, comprehensive research on this issue is contingent.

Previous researches have concentrated on creating differential models [16] and analyze the accuracy of their predictions [2]. For example, the SLIAR model is implemented for quick and accurate prediction [1], and the SIDARTHE model was designed to optimize the prediction in Italy [5]. Admittedly, decision tree algorithms are widely applied in biological and biomedical fields since it is useful for aggregating various types of data to make precise predictions on a single class [7]. The applications include medical decision making for precise diagnostic [10], gene microarray data analysis [9], as well as synthetic sick \& lethal genetic interactions [4]. However, the demographic datasets related to COVID-19 often involve multiple classes related to each other through a hierarchy, and each class can contain multiple labels. For example, the hierarchical classes for a human population dataset can be country, province, and city, while that for plant morphological data are the taxonomic ranks. Each higher-level hierarchy (e.g. country) includes the lower one (e.g. city). A more concrete example is China as a country includes Beijing, Shanghai, and so on as cities.

In this paper, the main point is to generate sets of similar countries defined by the small difference in infected, recovered, and dead time-series curves of the countries, taking the populationdemographic-COVID-19 (PDC) dataset as an input. The output set generated can be visualized graphically as a decision tree. Due to the hierarchical multi-label nature of the dataset, for which normal decision tree cannot handle, a novel sequential decision tree (SDT) algorithm for hierarchical multi-label classification is proposed (in section 2.2)

The SDT algorithm sequentially predicts each class label from the broader to narrower level: (a) It splits data points using criteria of entropy at the top level, (b) when the information gain is below 


\begin{tabular}{|c|c|c|c|c|c|}
\hline Attribute 1 & $\cdots$ & Attribute N & Class 1 & $\ldots$ & Class $\mathrm{C}$ \\
\hline value $1-1$ & $\ldots$ & value $1-\mathrm{N}$ & label 1-1 & $\ldots$ & label 1-M \\
\hline . & 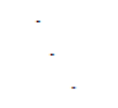 & . & . & $\cdot$ & . \\
\hline value $\mathrm{N}-1$ & $\ldots$ & value $\mathrm{N}-\mathrm{N}$ & label M-1 & $\ldots$ & label M-M \\
\hline
\end{tabular}

Figure 1: Data format

\begin{tabular}{|c|c|c|}
\hline Population-related & Demographic & COVID-19-related \\
\hline Urban population & Life expectancy (years) & $\begin{array}{l}\text { International travel controls } \\
\text { (OxBSG) }\end{array}$ \\
\hline Rural population & Literacy rate (\%) & Restrictions on gatherings (OxBSG) \\
\hline Total population & GDP per capita & Close public transport (OxBSG) \\
\hline Population Median Age & Political Regime (Score) & Stay at home requirements (OxBSG) \\
\hline \multirow[t]{2}{*}{$\begin{array}{l}\text { Population density (people per } \mathrm{km}^{2} \text { of land } \\
\text { area) }\end{array}$} & Share of deaths from unsafe sanitation (\%) & Residential (\%) \\
\hline & health care expenditure (\% of GDP) & \\
\hline
\end{tabular}

Figure 2: Attributes selected for SDT

a preset threshold, then it terminates at a level and switches to the lower level if it exists, otherwise making the current node a leaf node, and (c) repeat (a) and (b) recursively.

The main research goal in this paper is to compute the similarity of countries under the influence of COVID-19 using PDC as an input dataset. In summary, firstly, the most similar countries produced from the SDT algorithm are determined and grouped. Secondly, it is observed that the similarity is affected by geographic locations, meaning the countries on the same continent tend to be more similar. Finally, from data and statistical analysis, the hypothesis that most similar countries predicted from the PDC dataset, which is defined by the DT leaves branched from the same end node, also have similar COVID-19 evolutions, defined by the small difference in infected, recovered, and dead time series curves of the countries, was supported.

\section{DATA AND METHOD}

\subsection{SDT vs Clus-HMC}

Another known decision tree algorithm for hierarchical multi-label classification was introduced by Vens and his colleagues. Specifically, their study showed that the hierarchical multi-label classification (HMC) approach based on clustering outperforms single label (SC). The advantages of the clustering HMC (Clus-HMC) method include: (1) the class labels are predefined and can be represented as a tree, and (2) for each training example, the hierarchical class label can be represented as a numerical vector, so the reduction of Euclidian distance can be used as a criterion to split, which potentially reduce the time complexity for splitting comparing to the entropy criteria [15].

Nevertheless, the Clus-HMC method might not preserve the hierarchical orders at each level as the leaf will allow the impurity of class labels. Therefore, SDT is more applicable when the preservation of hierarchy in class labels is important. Specifically, even when leaves contain more than one class label at a level due to lack of deterministic attribute-values, the internal hierarchy of the labels is still preserved when using the SDT. For example, a leaf can contain multiple countries but all of them belong to the same subcontinent.

\subsection{Input Dataset for SDT}

Applying SDT requires a dataset with hierarchical classes and attributes. Define N, C, and M to be arbitrary positive integers. As shown in Figure 1, the data table is defined to contain $\mathrm{N}$ feature vectors and $C$ hierarchically structured classes, where each feature vector contains $\mathrm{N}$ values, and each class contains $\mathrm{M}$ labels. In addition, all numeric, categorical, and missing values are allowed for SDT.

For the COVID-19 application, numerous datasets were obtained from Our World in Data, 16 attributes relevant to COVID-19 were selected as shown in Figure 2, and a table with the feature vectors was made [7]. Also, 225 countries/regions, 23 subcontinents, and 5 continents were added to the table as class labels. For example, a row of class labels can be the United States ( $\epsilon$ class country), North 


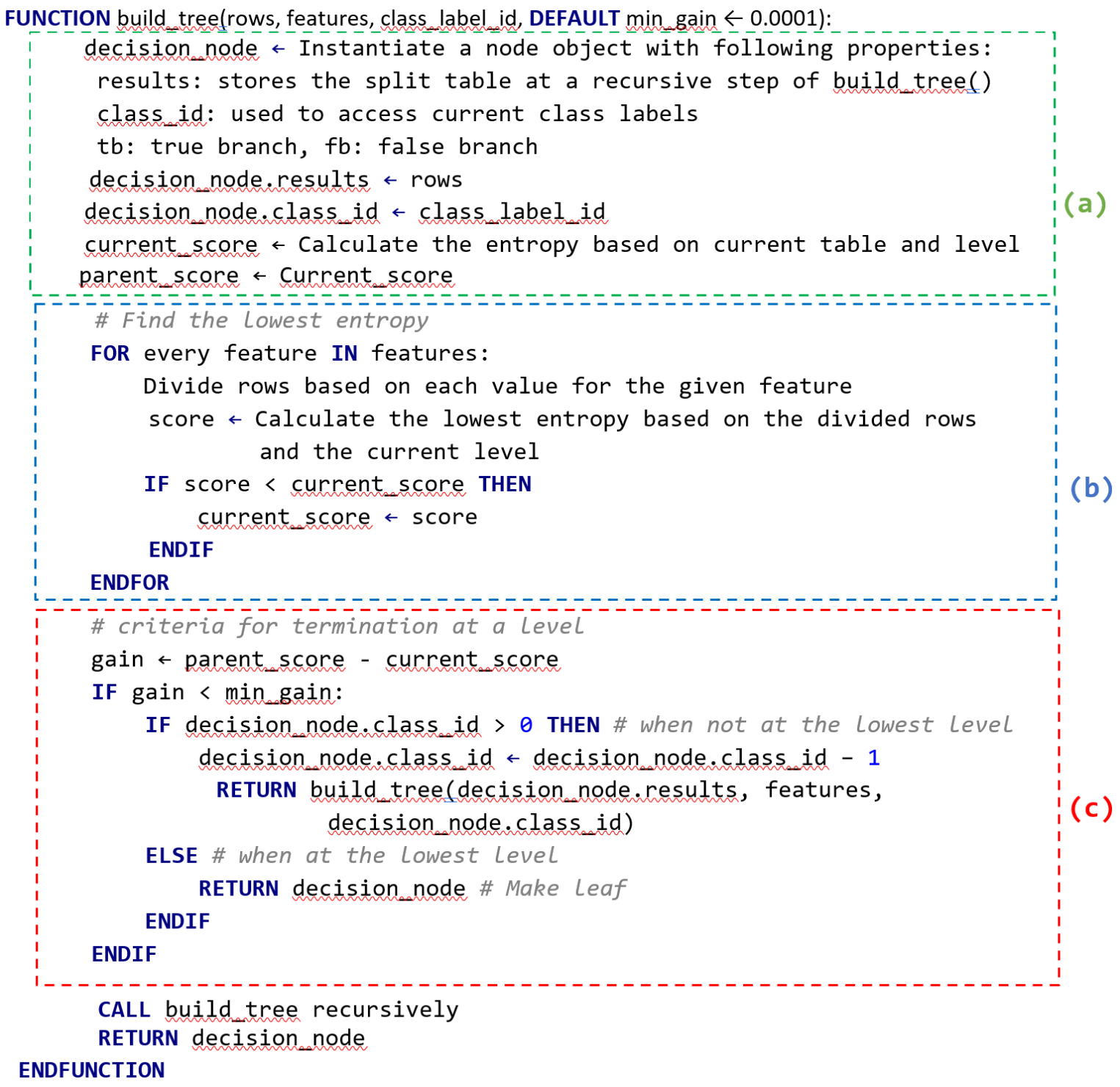

Figure 3: Pseudocode of SDT algorithm

America ( $\in$ class subcontinent), and America ( $\in$ class continent). The class labels, feature vectors are integrated to create a populationdemographic-COVID-19 (PDC) dataset as an input of SDT.

\subsection{Algorithmic Analysis}

The pseudocode for SDT is outlined in Figure 3 with each section color-coded and labeled. First, initialize the following variables: rows $\leftarrow$ READ file containing the table with values and labels, features $\leftarrow$ READ file header with all feature names, and class_label_id $\leftarrow$ the highest-level id that equals to the total number of class label -1 . The sections of the code are explained as follow: (a) initialize a tree node with the following properties: true and false branches (to reference to subtrees), results (to store the rows with values and class labels), class id (to keep track of the current level), (b) split data rows by the attribute-value with the lowest entropy, and (c) terminate at a level if no information is gained and switch to the lower level if it exists, otherwise make the current node a leaf node. Finally, build tree left and right recursively: decision_node.tb $\leftarrow$ build_tree(best_sets_t $\leftarrow$ true rows divided from the value with lowest entropy, features, decision_node.class_id), decision_node.fb $\leftarrow$ build_tree(best_sets_f $\leftarrow$ false rows divided from the value with lowest entropy, features, decision_node.class_id).

\subsection{Predictive Tool for COVID-19}

The PDC dataset was taken as an input, the SDT algorithm was used to build the tree. The countries in the leaves branched from the same node are defined to be the most similar. The hypothesis was made that the most similar countries predicted from the PDC 


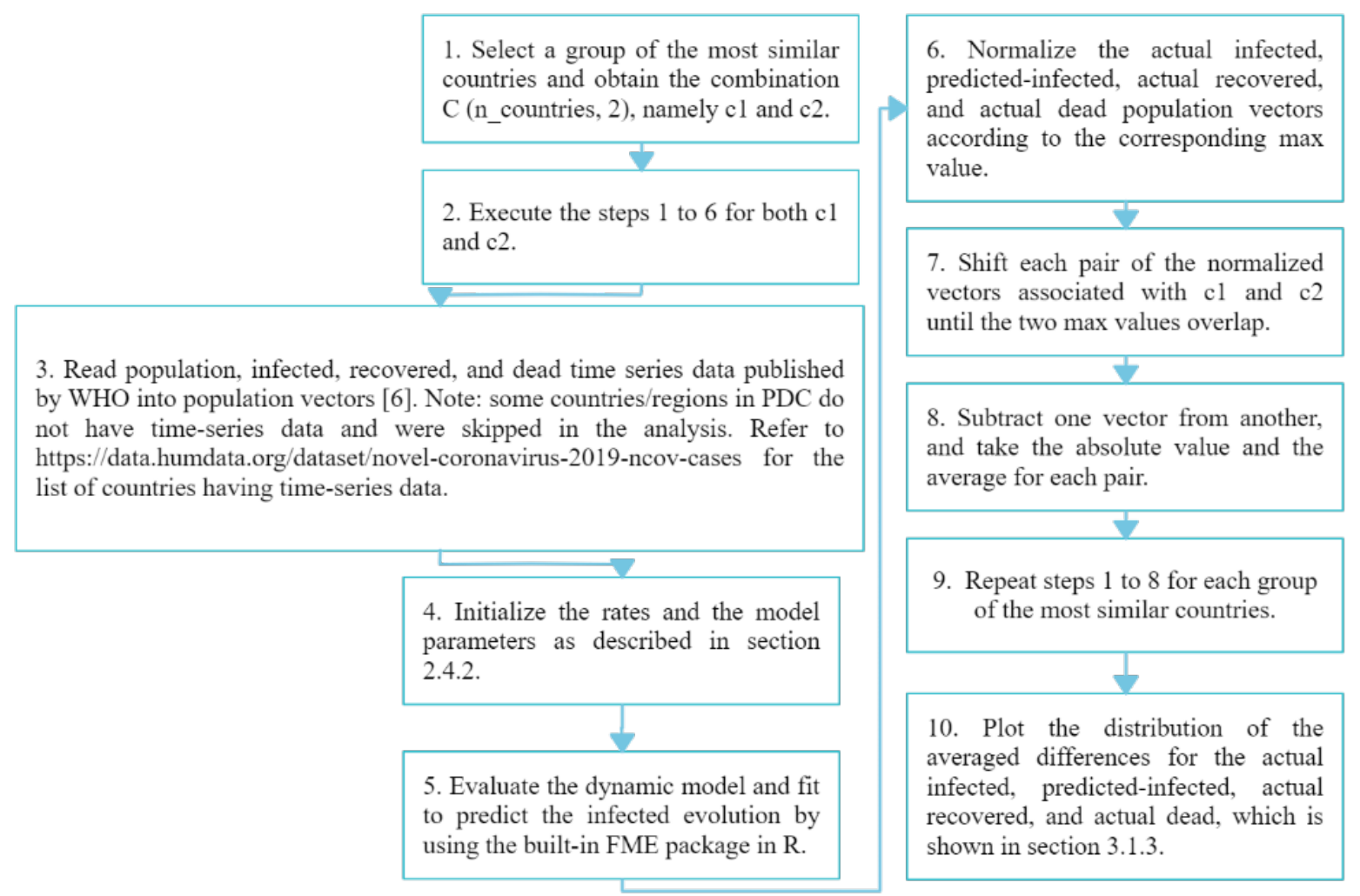

Figure 4: Flow chart showing the procedure for the similarity analysis.

dataset, which is defined by the DT leaves branched from the same end node, also have similar COVID-19 evolutions, defined by the small difference in infected, recovered, and dead time series curves of the countries.

2.4.1 Procedure for Similarity Analysis. The procedures for the Similarity Analysis are shown in Figure 4, which is performed after obtaining SDT results. Note if the hypothesis is supported, the COVID-19 evolutions of the skipped countries/regions can be simulated by their most similar counterparts having the time seriesdata.

2.4.2 Choice of Dynamic Model (SEIRD).. The dynamic model mentioned in step 5 described in Figure 4 is a system of ordinary differential equations. Specifically, combined with the SEIR model, a SEIRD model as shown in Figure 5 is implemented and applied to simulate the COVID-19 evolution [6]. S, E, I, R, and D stand for susceptible, exposed, infected, recovered, and dead, respectively, where E means infected but not yet infectious while I is infected and infectious. In terms of rates, $\mu$ represents natural birth and death rates. $\beta, \sigma$, and $\gamma$ are exposed, infected, and recovered rates, respectively, and $\alpha$ is the death rate due to the virus.

The parameters and rates are initialized as follows: $\alpha=1 \mathrm{e}-2$, $\beta=2 \mathrm{e}-9, \gamma=1 \mathrm{e}-6, \mu=1 \mathrm{e}-2, \sigma=1 \mathrm{e}-4, \mathrm{~S}=$ population of a country, $\mathrm{E}=1, \mathrm{I}=1, \mathrm{R}=0$, and $\mathrm{D}=0$. These initializations make sure the FME

$$
\begin{gathered}
\frac{d S}{d t}=\mu-\mu S-\beta S I \\
\frac{d E}{d t}=\beta S I-\mu E-\sigma E \\
\frac{d I}{d t}=\sigma E-\gamma I-\mu I-\alpha I \\
\frac{d R}{d t}=\gamma I-\mu R \\
\frac{d D}{d t}=\alpha I
\end{gathered}
$$

Figure 5: System of ODEs for the SEIRD model

package in $\mathrm{R}$ can perform gradient descent with appropriate starting values.

As shown in Figure 6, the model dynamics of the system of ODEs are plotted. Like the SIR model, the SEIRD model captures an inverse relationship between the susceptible, and infected populations. It is also worth noticing that the exposed population curve leads to the actually infected curve, and the recovered and dead populations start to increase when the I term becomes non-zero. 


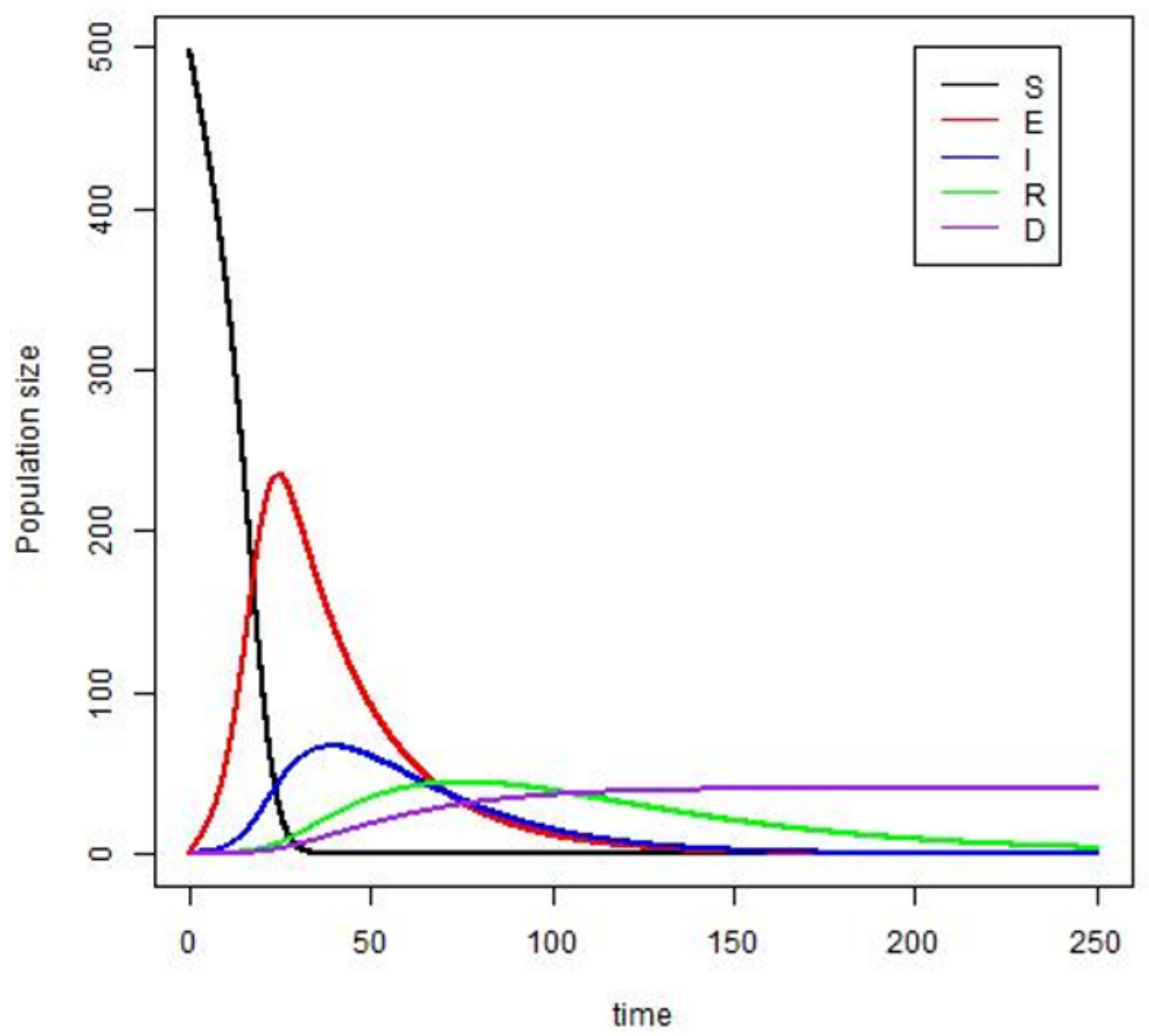

Figure 6: SEIRD model dynamics

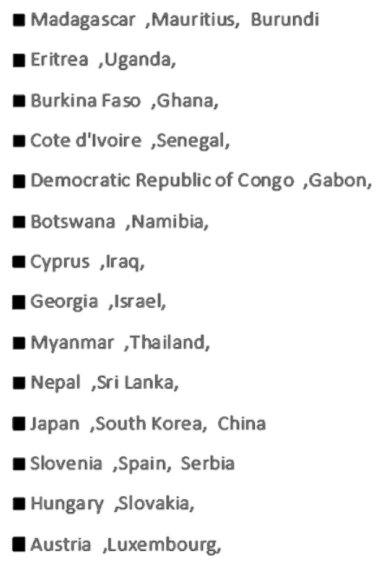

- Malawi ,Zambia,

Ethiopia ,Kenya,

- Benin ,Mali,

- Liberia ,Niger,

- Equatorial Guinea ,Sao Tome and Principe,

- South Africa, Swaziland, Lesotho

- Palestine, Turkey,

- Jordan ,Yemen,

- Singapore ,Timor, Cambodia

- Bhutan ,Maldives,

- Mongolia ,Taiwan, North Korea

- Malta ,Montenegro, Andorra

- Bulgaria ,Czech Republic, Belarus

- Finland, Sweden,
- Djibouti ,Tanzania,

- Seychelles ,Somalia,

- Nigeria ,Sierra Leone, Guinea

-Cameroon ,Congo, Angola

- Libya ,Tunisia, Algeria

- Kuwait ,Armenia,

- Lebanon ,United Arab Emirates,

- Laos, Vietnam,

- Bangladesh, Pakistan,

- Kazakhstan ,Tajikistan,

- Bosnia and Herzegovina ,Greece, Albania

- Russia ,Ukraine, Poland

- Netherlands, Switzerland,

- Iceland ,Lithuania, Denmark
- Mozambique ,Rwanda,

- Comoros, South Sudan,

- Mauritania ,Togo, Gambia

- Central African Republic ,Chad,

morocco, Sudan, Egypt

- Qatar ,Saudi Arabia, Oman

- Azerbaijan ,Bahrain,

- Malaysia ,Philippines, Indonesia

- Afghanistan ,India,

- Turkmenistan ,Uzbekistan, Kyrgyzstan

- Italy ,Portugal, Croatia

- Moldova ,Romania,

- France, Germany, Belgium

- Ireland ,United Kingdom, Estonia

Figure 7: Most similar country groups produced from SDT 


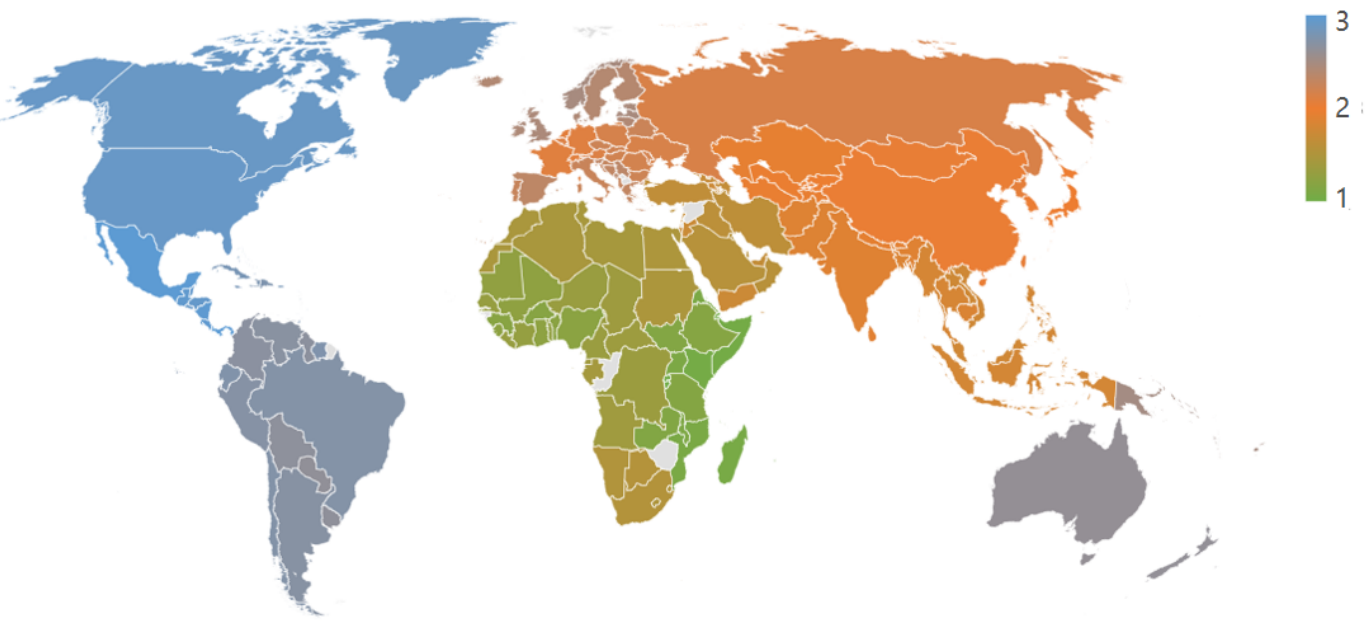

Figure 8: Map representation of COVID-19-based country similarity
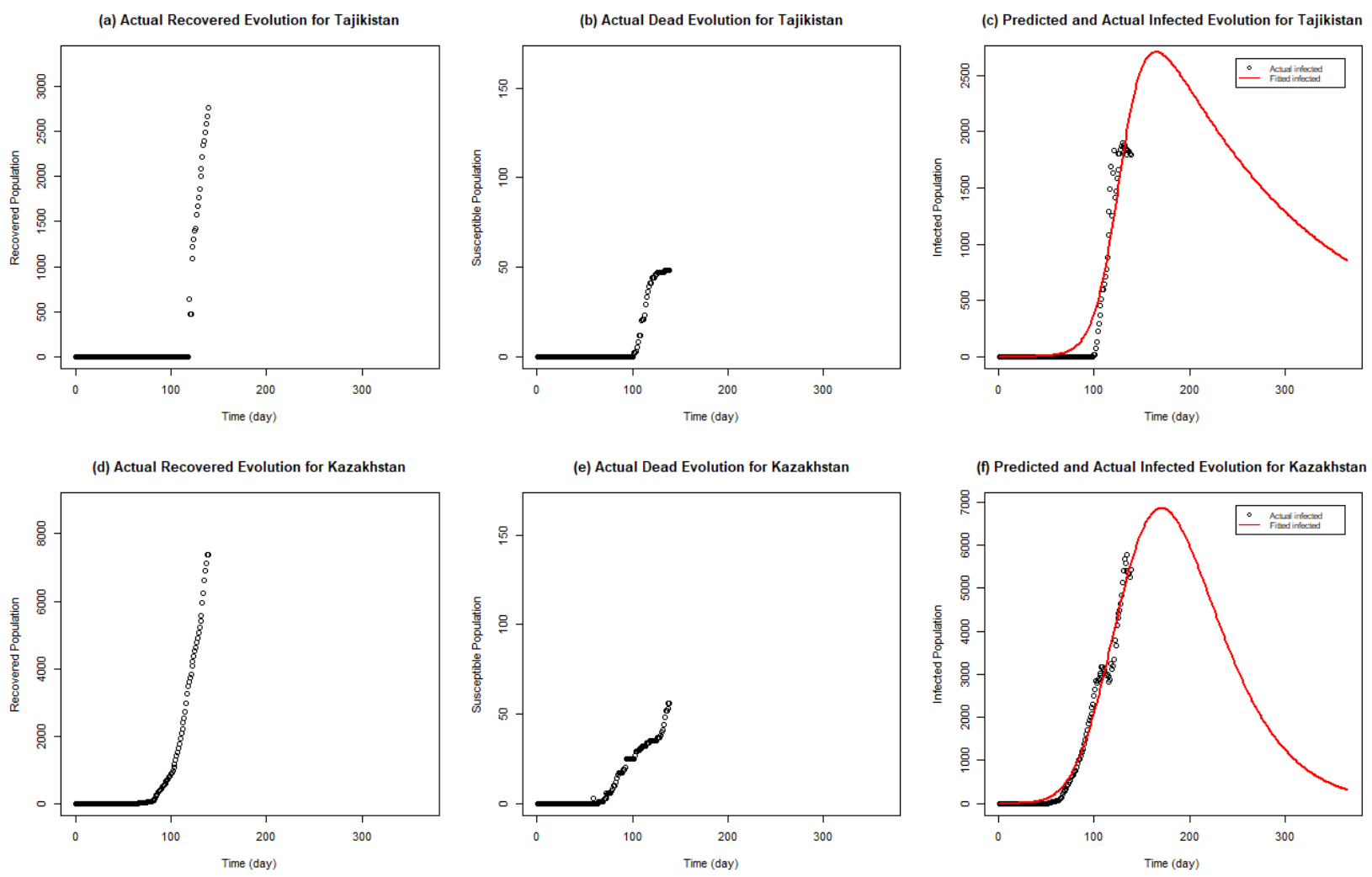

Figure 9: COVID-19 evolution curves for Tajikistan and Kazakhstan 

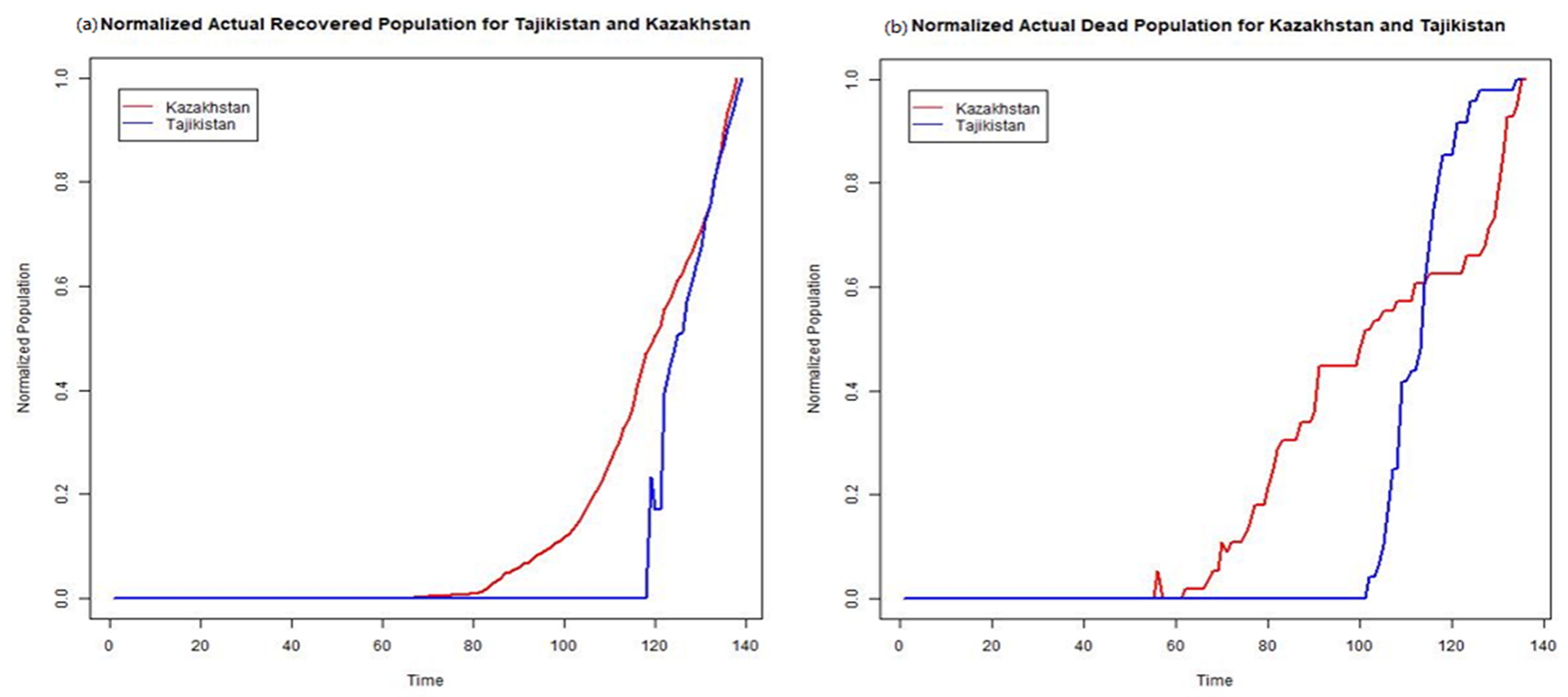

(c) Normalized Actual Infected Population for Kazakhstan and Tajikistan

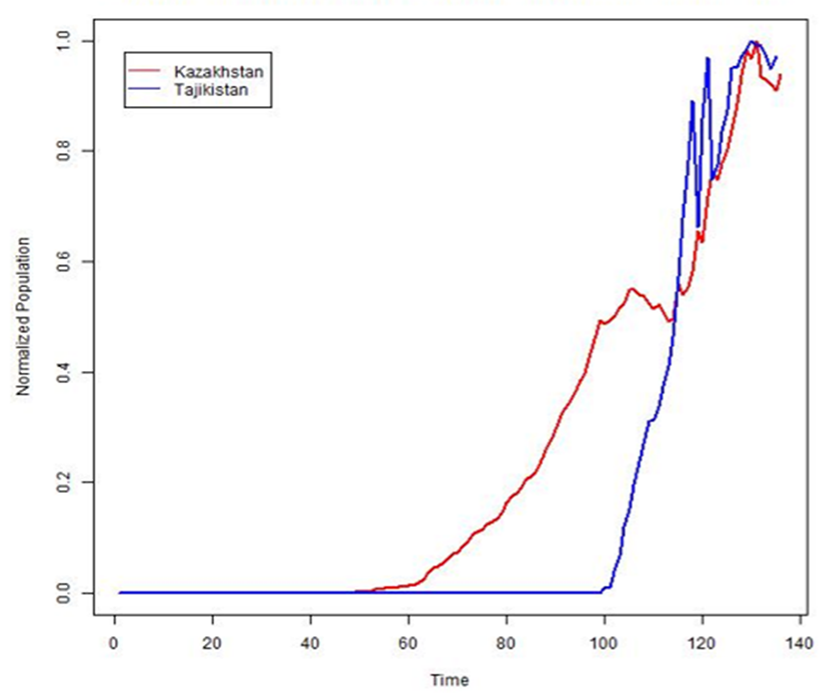

(d) Normalized Predicted Infected Population for Kazakhstan and Tajikistan

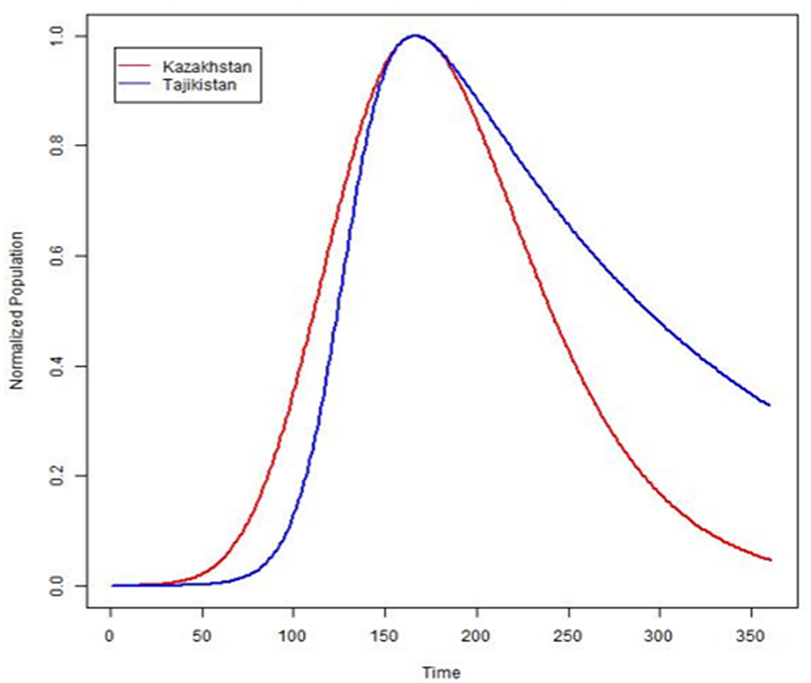

Figure 10: The time-shifted normalized- curves of COVID-19 evolution for Tajikistan and Kazakhstan

\section{RESULTS}

\subsection{SDT Classification}

As shown in Figure 7, it can be observed that the most similar countries produced from the SDT algorithm are grouped. Each group of countries represents the SDT leaves branched from the same node.

In addition, a map representation of the groups is plotted as shown in Figure 8. Specifically, 3 numbers represent 3 different levels of similarities: the closer the color, the more similar the countries. Also, it appears that the similarity is affected by geographic locations, meaning the countries on the same continent tend to be more similar. One potential reason is that the countries on the same continent tend to have similar demographic data (e.g. life expectancy, GDP per capita, and literacy rate as collected in the PDC dataset), leading the SDT algorithm to more likely to classify them into the same group [13]. It should also be noted that the 3 levels are very broad generalization for visualization purposes and might not associate with any statistical significance when performing the similarity analysis on the levels.

\subsection{Case Study}

Taking one group of the most similar countries, Tajikistan and Kazakhstan (row 10 column 3 in Figure 7), as an example, and performing the similarity analysis, it can be seen from Figure 9 that the evolutions of actual recovered, dead, and infected as well as the predicted infected appear to have similar shapes overall. 
(a) Actual Recovered

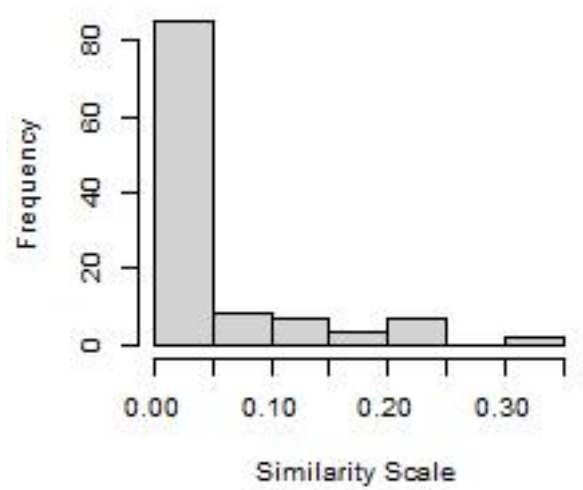

(c) Actual Infected

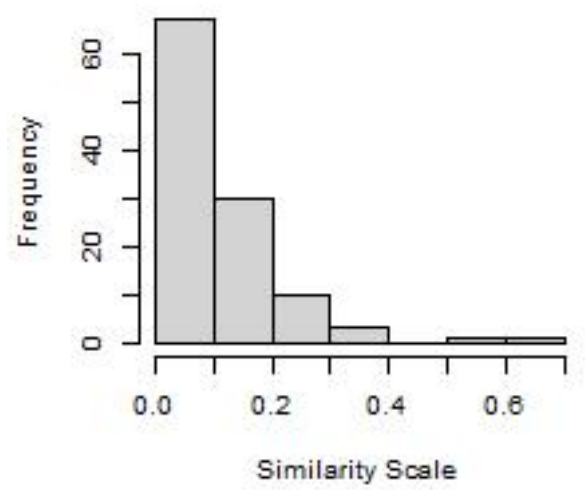

(b) Actual Dead

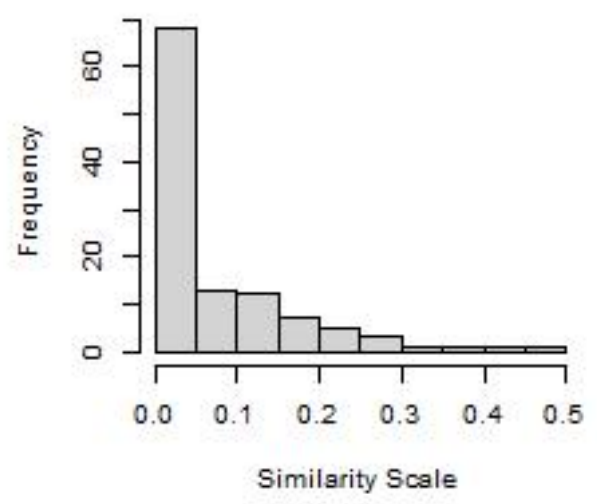

(d) Predicted Infected

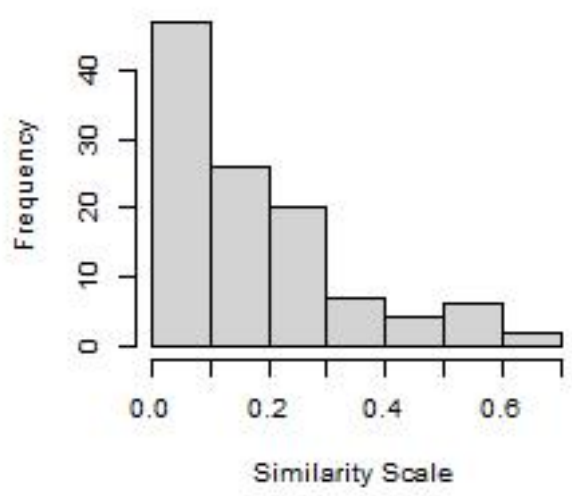

Figure 11: Distribution of COVID-19 curve differences

As shown in Figure 10, further plotting the time-shifted normalized disease curves for Tajikistan and Kazakhstan allows us to compare the curves' amplitudes and shapes. The COVID evolution patterns of the countries appear to be similar except the normalized actual dead curves in Figure 10 (b), which could be resulted from other factors not covered in the PDC dataset. From this pattern, it can be discovered that Tajikistan and Kazakhstan would potentially have similar COVID evolutions if the first case had occurred on the same day.

Finally, Figure 11 shows the overall distribution of the curve average differences between the most similar countries like Tajikistan and Kazakhstan discussed, meaning the similarity analysis in section 2.4.1 was performed on each group of the most similar countries. The difference is measured on a scale from 0 to 1 . A difference measure of 0 means two time-shifted curves completely overlap with the same amplitude, while 1 means the average amplitude of one curve is two times larger/smaller than that of the other curve. Overall, the similarity indices are mostly below 0.3 and the distributions in plots (a), (b), (c), (d) appear to be right-skewed.
Nevertheless, it appears that the predicted infected curves in plot (d) have a higher frequency of larger differences comparing to the actual ones.

\section{CONCLUSION}

In this paper, it was acknowledged that decision tree algorithm that handles single classes is widely used but the algorithm that can effectively perform hierarchical multiple classifications on the SDC dataset was not developed. As a result, the SDT algorithm for hierarchical multi-label classification was proposed, and the SDT application to COVID-19 was examined. Comparing to the existing algorithms, SDT has good interpretability to be used on the population-demographic-COVID-19 (PDC) dataset.

The main research goal in this paper is to compute the similarity of countries under the influence of COVID-19 using PDC as an input dataset. First, the most similar countries produced from the SDT algorithm were determined and grouped. Second, it was observed that the similarity is affected by geographic locations, meaning the countries on the same continent tend to be more similar. 
The objectives of this paper are to introduce the SDT algorithm, apply SDT to predict countries sharing most similarities related to COVID-19, and perform the similarity analysis to test whether the most similar countries have similar COVID-19 evolutions, including actual infection, recovery, death, as well as the predicted infection by using the SEIRD model. First, the overall similarity associated with closer geographic locations suggests the nature of SDT, which means that it classifies class labels sequentially from continent to country. Second, the right-skewed distributions shown in the result section indicates that the correlation exists between PDCbased groups of most similar countries and the groups' COVID-19 evolutions. Therefore, the hypothesis that the most similar countries predicted from the PDC dataset has similar COVID-19 evolutions is supported.

\section{ACKNOWLEDGMENTS}

The authors wish to thank Dr. Otto Cordero at the Parsons Laboratory, and Dr. Marina Barsky at Bard College at Simon's Rock, for helpful discussions during work on mathematical model and machine learning.

\section{REFERENCES}

[1] Arino, J., \& Portet, S. (2020, April 28). A simple model for COVID-19. Retrieved September 28, 2020, from https://www.sciencedirect.com/science/article/pii/ S2468042720300129

[2] Contreras, S., Villavicencio, H., Medina-Ortiz, D., Biron-Lattes, J., \& OliveraNappa, Á. (2020, May 25). A multi-group SEIRA model for the spread of COVID19 among heterogeneous populations. Retrieved September 28, 2020, from https: //www.sciencedirect.com/science/article/pii/S0960077920303246

[3] Geneva, S. (2020, August 5). Coronavirus disease. Retrieved September 28, 2020 from https://www.who.int/docs/default-source/coronaviruse/situation-reports/ 20200805-covid-19-sitrep-198.pdf?sfvrsn=f99d1754_2

[4] Geneva, S. (2020, July 15). Social Distancing, Quarantine, and Isolation. Retrieved September 28, 2020, from https://www.cdc.gov/coronavirus/2019-ncov/prevent- getting-sick/social-distancing.html

[5] Giordano, G., Blanchini, F., Bruno, R., Colaneri, P., Filippo, A., Matteo, A., \& Colaneri, M. (2020, April 22). Modeling the COVID-19 epidemic and implementation of population-wide interventions in Italy. Retrieved September 28, 2020, from https://www.nature.com/articles/s41591-020-0883-7

[6] Hethcote, H., Mathematics, D., Adler, P., Desmarchelier, S., Pinho, M., Bloodgood, J., . . . Dodd, C. (2019, August 21). THE BASIC EPIDEMIOLOGY MODELS: MODELS, EXPRESSIONS FOR R0, PARAMETER ESTIMATION, AND APPLICATIONS. Retrieved September 28, 2020, from https://www.worldscientific.com/doi/abs/10. 1142/9789812834836_0001

[7] Kingsford, C., \& Salzberg, S. (2008, September). What are decision trees? Retrieved September 28, 2020, from https://www.ncbi.nlm.nih.gov/pmc/articles/ PMC2701298

[8] Marindi, J. (2020, September 28). Novel Coronavirus (COVID-19) Cases Data. Retrieved September 28, 2020, from https://data.humdata.org/dataset/novelcoronavirus-2019-ncov-cases

[9] Polaka, I. (2010). Decision Tree Classifiers in Bioinformatics. Retrieved September 28, 2020, from https://www.researchgate.net/publication/220625930_Decision_ Tree_Classifiers_in_Bioinformatics

[10] Quinlan, J., C.. Shannon, W., A.. Paterson, T., J. T.. Tou, R., Murthy, K., R.. Neapolitan, K., . . . C. J.. Sims, L. (1993, January 01). Decision Trees: An Overview and Their Use in Medicine. Retrieved September 28, 2020, from https://link.springer.com/article/10.1023/A:1016409317640

[11] R. Lu, X., P. Zhou, X., XW. Xu, X., S. Sanche, Y., WG. Carlos, C., J. Riou, C., . . X. Qian, R. (1970, January 01). China's practice to prevent and control COVID-19 in the context of large population movement. Retrieved September 28, 2020, from https://idpjournal.biomedcentral.com/articles/10.1186/s40249-020-00716-0

[12] Rochester, M. (2020, September 11). Coronavirus disease 2019 (COVID-19). Retrieved September 28, 2020, from https://www.mayoclinic.org/diseasesconditions/coronavirus/symptoms-causes/syc-20479963

[13] Romualdi, C. (2002, April 12). Patterns of Human Diversity, within and among Continents, Inferred from Biallelic DNA Polymorphisms. Retrieved September 28, 2020, from http://europepmc.org/articles/PMC187513

[14] Roser, M. (2020, September 28). Our World in Data. Retrieved September 28, 2020,

from https://ourworldindata.org/
[15] S. F.. Altschul, T., Ashburner, M., Z.. Barutcuoglu, R., L.. Breiman, J., N.. CesaBianchi, C., S.. Chu, J., . . Y Yang, Y. (1997, January 01). Decision trees for hierarchical multi-label classification. Retrieved September 28, 2020, from https: //link.springer.com/article/10.1007/s10994-008-5077-3

[16] Smith, B. (2020, May 30). A novel: The impact of serial interval on a modifiedIncidence Decay and Exponential Adjustment (m-IDEA) model for projections of daily COVID-19 cases. Retrieved September 28, 2020, from https://www. sciencedirect.com/science/article/pii/S2468042720300166 